T. Yamaguchi

Nagoya Math. J.

Vol. 97 (1985), 173-192

\title{
ON THE NUMBER OF DIFFEOMORPHISM CLASSES IN A CERTAIN GLASS OF RIEMANNIAN MANIFOLDS
}

\author{
TAKAO YAMAGUCHI
}

\section{§ 0. Introduction}

The study of finiteness for Riemannian manifolds, which has been done originally by J. Cheeger [5] and A. Weinstein [13], is to investigate what bounds on the sizes of geometrical quantities imply finiteness of topological types, -e.g. homotopy types, homeomorphism or diffeomorphism classes- of manifolds admitting metrics which satisfy the bounds. For a Riemannian manifold $M$ we denote by $R_{M}$ and $K_{M}$ respectively the curvature tensor and the sectional curvature, by $\operatorname{Vol}(M)$ the volume, and by $\operatorname{diam}(M)$ the diameter.

Cheeger's finiteness theorem I [5]. For given $n, \Lambda, V>0$ there exist only finitely many pairwise non-diffeomorphic (non-homeomorphic) closed $n(\neq 4)$-manifolds (4-manifolds) which admit metrics such that $\left|K_{M}\right| \leq \Lambda^{2}$, $\operatorname{diam}(M) \leq 1, \operatorname{Vol}(M) \geq V$.

He proved directly finiteness up to homeomorphism for all dimension, and then for $n \neq 4$ used the results of Kirby and Siebenmann which show that finiteness up to homeomorphism implies finiteness up to diffeomorphism. For $n=4$, he put a stronger bound on $\|\nabla R\|$, where $\nabla R$ denotes the covariant derivative of curvature tensor $R$. For given $n, \Lambda, \Lambda_{1}, V>0$, we denote by $\mathfrak{M}^{n}\left(\Lambda, \Lambda_{1}, V\right)$ a class of closed $n$-dimensional Riemannian manifolds $M$ which satisfy the following bounds;

$$
\left|K_{M}\right| \leq \Lambda^{2}, \quad\left\|\nabla R_{M}\right\| \leq \Lambda_{1}, \quad \operatorname{diam}(M) \leq 1, \quad \operatorname{Vol}(M) \geq V,
$$

and set $\mathfrak{M}\left(\Lambda, \Lambda_{1}, V\right)=\bigcup_{n} \mathfrak{M}^{n}\left(\Lambda, \Lambda_{1}, V\right)$.

Cheeger's finiteness theorem II [5]. For given $n, \Lambda, \Lambda_{1}, V>0$, the number $\#_{\text {diff }} \mathfrak{M}^{n}\left(\Lambda, \Lambda_{1}, V\right)$ of diffeomorphism classes in $\mathfrak{M}^{n}\left(\Lambda, \Lambda_{1}, V\right)$ is finite.

In the proof of the Cheeger finiteness theorem and our results as

Received March 27, 1984. 
well, an estimate of the injectivity radius $i(M)$ of the exponential map on $M$ plays an important role. But since in his proof Ascoli's theorem is used essentially, it seems to us that it is impossible to bound the number $\#_{\text {diff }} \mathfrak{M}^{n}\left(\Lambda, \Lambda_{1}, V\right)$ explicitly from above by using the proof as in [5]. The main purpose of this paper is to show the existence of an upper bound for $\#_{\text {diff }} \mathfrak{M}\left(\Lambda, \Lambda_{1}, V\right)$ and express upper bounds for $\#_{\text {diff }} \mathfrak{M}^{n}\left(\Lambda, \Lambda_{1}, V\right)$ and $\#_{\text {diff }} \mathfrak{M}\left(\Lambda, \Lambda_{1}, V\right)$ explicitly in terms of a priori given constants. For a Riemannian manifold we denote by $d$ the distance function induced from the Riemannian metric.

We obtain the following theorems.

Theorem 1. For given $n, \Lambda, \Lambda_{1}, R>0$ there exist $\varepsilon_{1}=\varepsilon_{1}(n)>0, r_{1}=$ $r_{1}\left(n, \Lambda, \Lambda_{1}, R\right)>0$ such that if complete $n$-dimensional manifolds $M$ and $\bar{M}$ satisfy the following conditions, then $M$ is diffeomorphic to $\bar{M}$;

1) $\left|K_{M}\right|,\left|K_{\bar{M}}\right| \leq \Lambda^{2},\left\|\nabla R_{M}\right\|,\left\|\nabla R_{\bar{M}}\right\| \leq \Lambda_{1}, i(M), i(\bar{M}) \geq R$,

2) for some $r, r \leq r_{1}$, and $\varepsilon, \varepsilon \leq \varepsilon_{1}$, there exist $2^{-(n+8)} r$-dense and $2^{-(n+9)} r$-discrete subsets $\left\{p_{i}\right\} \subset M,\left\{q_{i}\right\} \subset \bar{M}$ such that the correspondence $p_{i} \rightarrow q_{i}$ is bijective and $(1+\varepsilon)^{-1} \leq d\left(q_{i}, q_{j}\right) / d\left(p_{i}, p_{j}\right) \leq 1+\varepsilon$ for all $p_{i}, p_{j}$ with $d\left(p_{i}, p_{j}\right) \leq 20 r$. $\varepsilon_{1}$ and $r_{1}$ can be written explicitly; e.g.

$$
\begin{aligned}
& \varepsilon_{1}=10^{-20}(n+1)^{-8}(n !)^{-2} 2^{-\left(2 n^{2}+41 n\right)}, \\
& r_{1}=\min \left\{R / 140, \varepsilon_{1} / 20 \Lambda, \sqrt[3]{10^{-3} n^{-5} 2^{-\left(\left(n^{2}+17 n\right) / 2\right)} \Lambda_{1}^{-1}},\left(10\left(2 n^{2} \Lambda^{2}+1\right)\right)^{-1}\right\} .
\end{aligned}
$$

For a metric space $X$ a subset $A$ is $\delta$-dense iff for any $x \in X, d(x, A)$ $<\delta$. A subset $A$ is $\delta$-discrete iff any two points of $A$ have the distance at least $\delta$.

Let $\omega_{n}$ denote the volume of the standard unit $n$-sphere. If we set $R=\min \left\{\pi / \Lambda,(n-1) V /\left(2 \omega_{n-2} e^{(n-1) \Lambda}\right)\right\}$, then $R$ gives a lower bound of the injectivity radii $i(M)$ for all $M$ in $\mathfrak{M}^{n}\left(\Lambda, \Lambda_{1}, V\right)$, and every $M$ in $\mathfrak{M}\left(\Lambda, \Lambda_{1}, V\right)$ has the dimension at most $n_{0}$, where $n_{0}=2 \max \left\{\left[\log \left(k^{k+2} / k ! V\right)\right], k\right\}+3$, $k=\left[\pi e^{2 \Lambda+1}\right]+1$, (§1. Lemma). Let $\varepsilon_{1}=\varepsilon_{1}(n), r_{1}=r_{1}\left(n, \Lambda, \Lambda_{1}, R\right)$ be as in Theorem 1.

THEOREM 2.

$$
\begin{aligned}
& \underset{\text { diff }}{\#} \mathfrak{M}^{n}\left(\Lambda, \Lambda_{1}, V\right) \leq\left(2^{2 n+17} / \varepsilon_{1} r_{1}^{2}\right)^{\left(N_{2}^{N_{0}}\right)+1} N_{0}, \\
& \underset{\text { diff }}{\#} \mathfrak{M}\left(\Lambda, \Lambda_{1}, V\right) \leq \sum_{n=0}^{n_{0}}\left(2^{2 n+17} / \varepsilon_{1} r_{1}^{2}\right)^{\left(N_{0}\right)+1} N_{\ni},
\end{aligned}
$$

where, $N_{0}=\left[e^{\Lambda(n-1)} /\left(\Lambda 2^{-(n+9)} r_{1}\right)^{n}\right]$. 
Here we descrive another application of Theorem 1. For a bi-Lipschitz map $f: X \rightarrow Y$ between two metric spaces $X$ and $Y$, set

$$
l(f):=\inf \left\{L ; L^{-1} \leq d(f(x), f(y)) / d(x, y) \leq L \text { for all } x, y \in X\right\} .
$$

Definition. Define $\rho(X, Y)$ by

$$
\left\{\begin{array}{l}
\inf \{\log l(f) ; f: X \rightarrow Y \text { is bi-Lipschitz map }\} \\
\infty \text { if any bi-Lipschitz map does not exist. }
\end{array}\right.
$$

It is clear that $\rho$ is symmetric and satisfies the triangle inequality. In the case $X$ and $Y$ are compact, Ascoil's theorem implies

$$
\rho(X, Y)=0 \text { iff } X \text { is isometric to } Y \text {. }
$$

For a positive integer $n$ we denote by $\mathfrak{U}^{n}$ a class of complete $n$-dimensional Riemannian manifolds $M$ with

$$
\left|K_{M}\right|<\infty, \quad\left\|\nabla R_{M}\right\|<\infty, \quad i(M)>0 .
$$

Of course $\mathfrak{U}^{n}$ contains all compact Riemannian manifolds of dimension $n$. Conversely, according to [7] every noncompact $n$-manifold admits a metric which belongs to the class $\mathfrak{U}^{n}$. A theorem of Shikata [12] states that there exists an $\varepsilon(n)>0$ depending only on $n$ such that if compact $n$ dimensional Riemannian manifolds $M$ and $N$ satisfy $\rho(M, N)<\varepsilon(n)$, then they are diffeomorphic. We do not know whether $\rho$ is distance on $\mathfrak{U}^{n}$, but can extend the Shikata theorem to the class $\mathfrak{Q}^{n}$. Let $\varepsilon_{1}=\varepsilon_{1}(n)$ be as in Theorem 1 again.

Corollary 3. If $M$ and $N \in \mathfrak{A}^{n}$ satisfy $\rho(M, N)<\log \left(1+\varepsilon_{1}\right)$, then they are diffeomorphic.

Recently M. Gromov [8], [9] states without giving detail of the proof that a similar result to Theorem 1 holds without the assumption for $\|\nabla R\|$. But our Theorem 1 is still valid for noncompact manifolds. However the assumption for $\|\nabla R\|$ is essential in the proof of our Theorem 1. Our proof is of course different from Gromov's one. The main tool of our proof is a technique of center of mass which is developed in [2].

The remainder of the paper is organized as follows: Assuming Theorem 1, the proofs of Theorem 2 and Corollary 3 are given in Section 1. Theorem 1 is proved in Section 2 - Section 4 .

The author wishes to thank Professor K. Shiohama for his advice and encouragement. 


\section{§1. Proofs of Theorem 2 and Corollary 3}

For a $\delta>0$, a system of points $\left\{x_{i}\right\}$ in a metric space $X$ is called a $\delta$-maximal system of $X$ if $\left\{x_{i}\right\}$ is maximal with respect to the property that the distance between any two of them is greater than or equal to $\delta$. $\left\{x_{i}\right\}$ is a $\delta$-maximal iff it is a $\delta$-dense and $\delta$-discrete subset. We show that there exists a $\delta$-maximal system of every Riemannian manifold $M$. Take a sequence $X_{i}$ of compact subsets of $M$ such that $\cup_{i} X_{i}=M$, $\stackrel{\circ}{X}_{i+1} \supset X_{i}$, where $\AA$ denotes the interior of a set $A$. We denote by $i\left(X_{k}\right)$ the infimum of the injectivity radius of the exponential map at points of $X_{k}$, and set $r_{k}:=\frac{1}{2} \min \left\{\delta, i\left(X_{k}\right)\right\}$. Take a $\delta$-maximal system $\left\{p_{i 1_{1 \leq i \leq N_{*}}^{1}}\right.$ of $X_{1}$. Notice that since the balls $B\left(p_{i}^{1}, r_{1}\right)$ have compact closure, they are contained in some $X_{k_{1}}$, and together with the fact that $B\left(p_{i}^{1}, r_{1}\right)$ are disjoint, this implies

$$
N_{1} \leq \operatorname{Vol}\left(X_{k_{1}}\right) / \min \operatorname{Vol}\left(B\left(p_{i}^{1}, r_{1}\right)\right) .
$$

By induction, it is possible to take a $\delta$-maximal system $\left\{p_{i}^{k}\right\}_{1 \leq i \leq N_{k}}$ of $X_{k}$ such that $p_{i}^{k}=p_{i}^{j}$ for every $j<k$ and every $i, 1 \leq i \leq N_{j}$. Then the system $\bigcup_{k=1}^{\infty}\left\{p_{i}\right\}_{N_{k-1}+1 \leq i \leq N_{k}}$ is a $\delta$-maximal system of $M$, where $N_{0}:=0$.

Proof of Corollary 3 assuming Theorem 1. By the assumption there exists a bi-Lipschitz map $f: M \rightarrow N$ such that $l(f)<1+\varepsilon_{1}(n)$. We may assume

$$
\left|K_{M}\right|,\left|K_{N}\right| \leq \Lambda^{2},\left\|\nabla R_{M}\right\|,\left\|\nabla R_{N}\right\| \leq \Lambda_{1}, \quad i(M), i(N) \geq R,
$$

for some $\Lambda, \Lambda_{1}, R>0$. Let $r_{1}=r_{1}\left(n, \Lambda, \Lambda_{1}, R\right)$ be as in Theorem 1 , and take a $\left(1+\varepsilon_{1}\right) 2^{-(n+9)} r_{1}$-maximal system $\left\{p_{i}\right\}$ of $M$. Since $f$ is bi-Lipschitz, it is surjective. Therefore it is easy to show that $\left\{f\left(p_{i}\right)\right\}$ is $2^{-(n+8)} r_{1}$-dense and $2^{-(n+9)} r_{1}$-discrete.

Q.E.D.

To prove Theorem 2 we recall an injectivity radius estimate. From now on, for given $n$ and $\delta>0$, let $v(\delta)$ (resp. $\tilde{v}(\delta)$ ) denote the volume of a $\delta$-ball in the $n$-dimensional hyperbolic space with constant curvature $-\Lambda^{2}$ (resp. $n$-sphere with constant curvature $\Lambda^{2}$ ). The following lemma is a dimension independent version of [5], [10] and [11].

Lemma. For given $\Lambda, V>0$, there exist $n_{0}=n_{0}(\Lambda, V)$ and $R_{0}=R_{0}(\Lambda, V)$ $>0$ such that if $M$ is an n-dimensional compact Riemannian manifold such that $\left|K_{M}\right| \leq \Lambda^{2}, \operatorname{diam}(M) \leq 1, \operatorname{Vol}(M) \geq V$, then 
(1) $n=\operatorname{dim} M \leq n_{0}$,

(2) $i(M) \geq \min \left\{\pi / \Lambda,(n-1) V /\left(2 \omega_{n-2} e^{(n-1) \Lambda}\right)\right\} \geq R_{0}$,

where $n_{0}$ and $R_{0}$ can be written explicitly as

$$
\begin{aligned}
& n_{0}=2 \max \left\{\left[\log \left(k^{k+2} / k ! V\right)\right], k\right\}+3, \quad k=\left[\pi e^{2 \Lambda+1}\right]+1, \\
& R_{0}=\min _{2 \leq n \leq n_{0}}\left\{\pi / \Lambda,(n-1) V /\left(2 \omega_{n-2} e^{(n-1) \Lambda}\right)\right\} .
\end{aligned}
$$

Proof. For (1), the Rauch comparison theorem yields

$$
V \leq \operatorname{Vol}(M) \leq v(1) \leq \omega_{n-1} e^{(n-1) A},
$$

where

$$
\omega_{n-1}= \begin{cases}2 \pi^{m} /(m-1) ! & (n=2 m) \\ 2(2 \pi)^{m} /(2 m-1)(2 m-3) \cdots 3 \cdot 1 & (n=2 m+1) .\end{cases}
$$

Notice that

$$
\lim _{n \rightarrow \infty} \omega_{n-1} e^{(n-1) A}=0 .
$$

It is an easy calculation to estimate such an $n_{0}$ that $\omega_{n-1} e^{(n-1) \Lambda}<V$ for all $n>n_{0}$. For (2), it suffices to bound the lengths of closed geodesics from below. Suppose that there is a closed geodesic with length $l$. The Rauch comparison theorem implies that $\operatorname{Vol}(M)$ is not greater than the volume of the tublar neighborhood of radius 1 of a geodesic segment with length $l$ in the $n$-dimensional hyperbolic space with constant curvature $-\Lambda^{2}$. Therefore we get

$$
\begin{aligned}
\operatorname{Vol}(M) & \leq l \cdot \omega_{n-2} \int_{0}^{1}(\sinh \Lambda t / \Lambda)^{n-2} \cosh \Lambda t d t \\
& =l \cdot \omega_{n-2}(\sinh \Lambda)^{n-1} /(n-1) \Lambda^{n-1} \\
& \leq l \cdot \omega_{n-2} e^{(n-1) \Lambda} /(n-1) .
\end{aligned}
$$

Hence $l \geq(n-1) V /\left(\omega_{n-2} e^{(n-1) \Lambda}\right)$, and this yields (2).

Q.E.D.

Proof of Theorem 2 assuming Theorem 1. For each $M_{\alpha} \in \mathfrak{M}^{n}\left(\Lambda, \Lambda_{1}, V\right)$, take a $2^{-(n+8)} r_{1}$-maximal system $\left\{p_{i}^{\alpha}\right\}_{i}$ of $M_{\alpha}$. Note that since $\operatorname{diam}\left(M_{\alpha}\right) \leq 1$,

$$
\#\left\{p_{i}^{\alpha}\right\}_{i} \leq v(1) / \tilde{v}\left(2^{-(n+9)} r_{1}\right) \leq\left[e^{(n-1) \Lambda /}\left(\Lambda 2^{-(n+9)} r_{1}\right)^{n}\right]=N_{0} .
$$

Set $m:=\#_{\text {diff }} \mathfrak{M}^{n}\left(\Lambda, \Lambda_{1}, V\right), L:=1 /\left(2^{-(n+8)} r_{1}\right)$ and $\varepsilon_{1}^{\prime} ;=\varepsilon_{1} /\left(2\left(1+\varepsilon_{1}\right) L\right)$. Suppose that

$$
m>\left(2^{2 n+17} / \varepsilon_{1} r_{1}^{2}\right)^{\left(N_{0}\right)+1} N_{0}>\left(\left[L / 2 \varepsilon_{1}^{\prime}\right]+1\right)^{\left(N_{2}^{N_{0}}\right)+1} N_{0}
$$


Then $\mathfrak{M}^{n}\left(\Lambda, \Lambda_{1}, V\right)$ contains at least $\left[m / N_{0}\right]$ pairwise non-diffeomorphic manifolds $\left\{M_{\alpha}\right\}_{\alpha \in A}$ with the $2^{-(n+8)} r_{1}$-maximal systems whose numbers are all the same, say $N_{1}, N_{1} \leq N_{0}$. We concider the set

$$
\Sigma:=\left\{\left(i_{k}, j_{k}\right) ; 1 \leq k \leq\left(\begin{array}{l}
N_{1} \\
2
\end{array}\right):=N_{1}^{\prime}\right\}
$$

of all the distinct pairs of the indices of the systems $\left\{p_{i}^{\alpha}\right\}_{i}$ for $\left\{M_{\alpha}\right\}_{\alpha \in A}$. For each $M_{\alpha}$ and $M_{\beta}(\alpha, \beta \in A)$, and for each $\left(i_{k}, j_{k}\right) \in \Sigma$, we set $l(\alpha, \beta ; k)=$ $d\left(p_{i_{k}}^{\beta}, p_{j_{k}}^{\beta}\right) / d\left(p_{i_{k}}^{\alpha}, p_{j_{k}}^{\alpha}\right)$. Notice that $L^{-1} \leq l(\alpha, \beta ; k) \leq L$. We fix some $\alpha \in A$. For $\left(i_{1}, j_{1}\right) \in \Sigma$ there is a $t_{1} \in\left[L^{-1}, L\right]$ such that if

$$
A_{1}:=\left\{\beta \in A ; l(\alpha, \beta ; 1) \in\left[t_{1}-\varepsilon_{1}^{\prime}, t_{1}+\varepsilon_{1}^{\prime}\right]\right\}
$$

then $\# A_{1} \geq\left[m / N_{0}\right]\left(\left[L / 2 \varepsilon_{1}^{\prime}\right]+1\right)^{-1}$. By induction, for $\left(i_{k}, j_{k}\right) \in \Sigma$ there is a $t_{k} \in\left[L^{-1}, L\right]$ such that if

$$
A_{k}:=\left\{\beta \in A_{k-1} ; l(\alpha, \beta ; k) \in\left[t_{k}-\varepsilon_{1}^{\prime}, t_{k}+\varepsilon_{1}^{\prime}\right]\right\}
$$

then $\# A_{k} \geq\left[m / N_{0}\right]\left(\left[L / 2 \varepsilon_{1}^{\prime}\right]+1\right)^{-k}$. By the assumption on $m$, it is possible to take distinct pair $\beta$ and $\beta^{\prime}$ in $A_{N_{1}^{\prime}}$. Then $\left|l(\alpha, \beta ; k)-l\left(\alpha, \beta^{\prime} ; k\right)\right| \leq 2 \varepsilon_{1}^{\prime}$ for all $k, 1 \leq k \leq N_{1}^{\prime}$, and this implies $\left(1+\varepsilon_{1}\right)^{-1} \leq l\left(\beta, \beta^{\prime} ; k\right) \leq 1+\varepsilon_{1}$. This is a contradiction since by Theorem $1 M_{\beta}$ is diffeomorphic to $M_{\beta^{\prime}}$. The estimate for $\#_{\text {diff }} \mathfrak{M}\left(\Lambda, \Lambda_{1}, V\right)$ is an immediate consequence of the previous lemma (1) and the estimate for $\#_{\text {diff }} \mathfrak{M}^{n}\left(\Lambda, \Lambda_{1}, V\right)$.

Q.E.D.

\section{§. Construction of local diffeomorphisms}

The rest of this paper is devoted to the proof of Theorem 1. For given $n, \Lambda, R>0$, set $R_{0}:=\frac{1}{2} \min \{R, \pi / \Lambda\}$ and let $r$ and $\varepsilon$ be adjustable parameters with $0<r \leq R_{0} / 70,0<\varepsilon \leq 2^{-(n+14)}$. From now on we denote by $M$ and $\bar{M}$ complete $n$-dimensional Riemannian manifolds which satisfy the conditions in Theorem 1 for $r$ and $\varepsilon$. In the final part of the proof, we will set $r \leq r_{1}$, and $\varepsilon \leq \varepsilon_{1}$. We use the bound for $\|\nabla R\|$ actually only in Section 4. Let $\left\{p_{i}\right\} \subset M$ and $\left\{q_{i}\right\} \subset \bar{M}$ be $2^{-(n+8)} r$-dense and $2^{-(n+9)} r$-discrete subsets as in Theorem 1. For given $p \in M$ and $\delta>0$, we denote by $M_{p}$ the tangent space of $M$ at $p$, and by $B(p, \delta)$ the $\delta$-ball with center $p$. Note that all $\delta$-balls with $\delta \leq R_{0}$ in $M$ and $\bar{M}$ are convex and that by the Rauch comparison theorem, for any $v, w \in M_{p}$ with $\|v\|,\|w\| \leq t, t \leq R_{0}$

$$
\sin \Lambda t / \Lambda t \leq d\left(\exp _{p} v, \exp _{p} w\right) /\|v-w\| \leq \sinh \Lambda t / \Lambda t .
$$

The purpose of this section is to prove the following lemma. 
Lemma 2.1. For each $i$ there exists a linear isometry $I_{i}$ from $M_{p_{i}}$ to $\bar{M}_{q_{i}}$ such that if $F_{i}:=\exp _{q_{i}} \circ I_{i} \circ \exp _{p_{i}}^{-1}: B\left(p_{i}, R_{0}\right) \rightarrow B\left(q_{i}, R_{0}\right)$, then $d\left(F_{i}\left(p_{j}\right), q_{j}\right)$ $\leq \delta_{1} r$ for every $p_{j}$ with $d\left(p_{i}, p_{j}\right) \leq 10 r$, where

$$
\delta_{1}=2(n+1)\left(6^{n+2} n ! 2^{(n / 2)+7}\right)^{1 / 2}(40 A r+2 \varepsilon)^{1 / 4} .
$$

Proof. Set $\tilde{p}_{j}:=\exp _{p_{i}}^{-1}\left(p_{j}\right)$ and $\tilde{q}_{j}:=\exp _{q_{i}}^{-1}\left(q_{j}\right)$. Then $\left\{\tilde{p}_{j}\right\}$ and $\left\{\tilde{q}_{j}\right\}$ are $2^{-(n+7)} r$-dense and $2^{-(n+10)} r$-discrete subsets of the $10 r$-ball around 0 and satisfy $(1+\varepsilon)^{-1} e^{-2011} \leq\left\|\tilde{q}_{j}-\tilde{q}_{k}\right\| /\left\|\tilde{p}_{j}-\tilde{p}_{k}\right\| \leq(1+\varepsilon) e^{201 r}$ for all $j, k, j \neq k$. Hence Lemma 2.1 is a direct consequence of the following.

Lemma $2.1^{\prime}$. Let $\left\{x_{i}\right\}$ be a $2^{-(n+7)} r$-dense and $2^{-(n+10)} r$-discrete subset of $B(0, r) \subset \boldsymbol{R}^{n}$ with $x_{1}=0$. If a system $\left\{y_{i}\right\}$ of points in $B(0, r)$ with $y_{1}=0$ satisfies $(1+\varepsilon)^{-1} \leq\left\|y_{i}-y_{j}\right\| /\left\|x_{i}-x_{j}\right\| \leq 1+\varepsilon$ for every $i \neq j$. Then there exists a linear isometry $I$ of $R^{n}$ such that

$$
\left\|I\left(x_{i}\right)-y_{i}\right\| \leq(n+1)\left(6^{n+2} \cdot n ! \cdot 2^{(n / 2)+7} \cdot \varepsilon^{1 / 2}\right)^{1 / 2} r
$$

for every $i$.

For the proof of the Lemma $2.1^{\prime}$, it is convenient to introduce the following notion, a normal system, and to investigate some properties of a normal system. This is done in Lemma 2.3-Lemma 2.5.

Definition 2.2. For $0 \leq \eta<1$ and $r>0$, we say that a system of $n$ points $\left\{p_{i}\right\}_{1 \leq i \leq n}$ of $\boldsymbol{R}^{n}$ is $(r, \eta)$-normal if $(1-\eta) r \leq\left\|p_{i}\right\| \leq r,\left|\left\langle p_{i}, p_{j}\right\rangle\right| \leq \eta r^{2}$ for every $i \neq j$.

Lemma 2.3. For every $L \geq n+1$, let $\left\{p_{i}\right\}_{1 \leq i \leq n}$ be an $\left(r, 2^{-L}\right)$-normal system for $\boldsymbol{R}^{n}$. If we set $p_{i}^{\prime}:=p_{i}-\left\langle p_{i}, u_{1}\right\rangle u_{1}-\cdots-\left\langle p_{i}, u_{i-1}\right\rangle u_{i-1}, u_{i}:=$ $p_{i}^{\prime} /\left\|p_{i}^{\prime}\right\|$ inductively, then

(1) $\left\|p_{i}^{\prime}\right\| \geq\left(1-2^{-(L-i)}\right)^{1 / 2} r \geq\left(1-2^{-(L-i)}\right) r$,

(2) $\left|\left\langle p_{k}, u_{i}\right\rangle\right| \leq 2^{-(L-i)} r$

for every $i, k$ with $k>i$.

Proof. For $i=1,(1)$ and (2) are trivial. Assume (1), (2) for $j, 1 \leq j$ $\leq i$. Then we get

$$
\begin{aligned}
\left\|p_{i+1}^{\prime}\right\|^{2} & =\left\|p_{i+1}\right\|^{2}-\left\langle p_{i+1}, u_{1}\right\rangle^{2}-\cdots-\left\langle p_{i+1}, u_{i}\right\rangle^{2} \\
& \geq\left(\left(1-2^{-L}\right)^{2}-2^{-2(L-1)}-\cdots-2^{-2(L-i)}\right) r^{2} \\
& \geq\left(1-2^{-(L-i-1)}\right) r^{2} \geq\left(1-2^{-(L-i-1)}\right)^{2} r^{2},
\end{aligned}
$$

and for $k>i+1$, 


$$
\begin{aligned}
\left|\left\langle p_{k}, u_{i+1}\right\rangle\right| \leq & \left\|p_{i+1}^{\prime}\right\|^{-1}\left(\left|\left\langle p_{k}, p_{i+1}\right\rangle\right|+\left|\left\langle p_{i+1}, u_{1}\right\rangle\right|\left|\left\langle p_{k}, u_{1}\right\rangle\right|+\cdots\right. \\
& \left.+\left|\left\langle p_{i+1}, u_{i}\right\rangle\right|\left|\left\langle p_{k}, u_{i}\right\rangle\right|\right) \\
\leq & 2\left(2^{-L}+2^{-2(L-1)}+\cdots+2^{-2(L-i)}\right) r \leq 2^{-L+i+1} r .
\end{aligned}
$$

Thus for $L \geq n+1$, the Gram-Schmidt orthonormalization procedure yields the orthonormal basis $\left\{u_{i}\right\}$ for $\boldsymbol{R}^{n}$ via an $\left(r, 2^{-L}\right)$-normal system $\left\{p_{i}\right\}$.

LEMMA 2.4. If $\left\{p_{i}\right\}_{1 \leq i \leq n}$ is an $\left(r, 2^{-L}\right)$-normal system for $\boldsymbol{R}^{n}$, and if for some $\delta>0, x$ and $y$ in $\boldsymbol{R}^{n}$ satisfy

$$
\|x\|,\|y\| \leq r, \quad|\|x\|-\|y\|| \leq \delta, \quad\left|\left\|x-p_{i}\right\|-\left\|y-p_{i}\right\|\right| \leq \delta
$$

for all $i, 1 \leq i \leq n$, then $\|x-y\| \leq 3\left(n+2^{-L+n+4}\right) \delta$.

Proof. Notice that

$$
\left|\left\langle p_{i}, x-y\right\rangle\right|=2^{-1}\left|\|x\|^{2}-\|y\|^{2}+\left\|p_{i}-y\right\|^{2}-\left\|p_{i}-x\right\|^{2}\right| \leq 3 \delta r .
$$

By induction, we show that

$$
\left|\left\langle u_{i}, x-y\right\rangle\right| \leq 3\left(1+2^{-L+i+1}\right)^{2} \delta .
$$

This is trivial for $i=1$. Assume $(*)$ for $j, 1 \leq j \leq i$. Then we have with Lemma 2.3

$$
\begin{aligned}
\left|\left\langle u_{i+1}, x-y\right\rangle\right| \leq & \left\|p_{i+1}^{\prime}\right\|^{-1}\left(\left|\left\langle p_{i+1}, x-y\right\rangle\right|+\left|\left\langle p_{i+1}, u_{1}\right\rangle\right|\left|\left\langle u_{1}, x-y\right\rangle\right|+\cdots\right. \\
& \left.+\left|\left\langle p_{i+1}, u_{i}\right\rangle\right|\left|\left\langle u_{i}, x-y\right\rangle\right|\right) \\
\leq & 3\left(1-2^{-L+i+1}\right)^{-1}\left(1+2^{-(L-1)}\left(1+2^{-L+2}\right)^{2}+\cdots\right. \\
& \left.+2^{-(L-i)}\left(1+2^{-L+i+1}\right)^{2}\right) \delta \\
\leq & 3\left(1+2^{-L+i+2}\right)\left(1+2^{-L+2}+\cdots+2^{-L+i+1}\right) \delta \\
\leq & 3\left(1+2^{-L+i+2}\right)^{2} \delta .
\end{aligned}
$$

Hence we conclude that

$$
\|x-y\| \leq \sum_{1}^{n}\left|\left\langle u_{i}, x-y\right\rangle\right| \leq \sum_{1}^{n} 3\left(1+2^{-L+i+1}\right)^{2} \delta \leq 3\left(n+2^{-L+n+4}\right) \delta .
$$

LEMma 2.5. For $k, 1 \leq k \leq n$, and $L \geq k+2$, let $\left\{e_{i}\right\}_{1 \leq i \leq k} \subset \boldsymbol{R}^{n}$ be $a$ $\left(1,2^{-L}\right)$-normal system for the linear subspace spanned by $\left\{e_{i}\right\}$ with $\left\|e_{i}\right\|=1$ for all $i$. If two unit vectors $x$ and $y$ which belong to Span $\left\{e_{i}\right\}_{1 \leq i \leq k}$ satisfy the following inequalities;

$$
\left|\Varangle\left(e_{i}, x\right)-\Varangle\left(e_{i}, y\right)\right| \leq \alpha \quad(1 \leq i \leq k-1),\left\langle x, e_{k}\right\rangle \geq 3 / 4,\left\langle y, e_{k}\right\rangle \geq 3 / 4,
$$


then $\Varangle(x, y) \leq 6\left((k-1)+2^{-L+k+3}\right) \alpha$, where $\Varangle(x, y)$ denotes the angle between $x$ and $y$.

Proof. Notice that $\left|\left\langle e_{i}, x\right\rangle-\left\langle e_{i}, y\right\rangle\right| \leq \alpha(1 \leq i \leq k-1)$, and

$$
2^{-1} \Varangle(x, y) \leq \sin \Varangle(x, y) \leq\|x-y\| \text {. }
$$

Hence it suffices to estimate $\|x-y\|$ from above. Let $\left\{u_{i}\right\}$ be an orthonormal basis for Span $\left\{e_{i}\right\}$ obtained by the Gram Schmidt process from $\left\{e_{i}\right\}$. From Lemma $2.4(*)$, we get $\left|\left\langle u_{i}, x-y\right\rangle\right| \leq\left(1+2^{L+i+1}\right)^{2} \alpha(1 \leq i \leq k-1)$. By Lemma 2.3,

$$
\begin{aligned}
\left\langle u_{k}, x\right\rangle & \geq\left\|e_{k}^{\prime}\right\|^{-1}\left(\left\langle e_{k}, x\right\rangle-\left|\left\langle e_{k}, u_{1}\right\rangle\left\|\left\langle u_{1}, x\right\rangle|-\cdots-|\left\langle e_{k}, u_{k-1}\right\rangle\right\|\left\langle u_{k-1}, x\right\rangle\right|\right) \\
& \geq\left\langle e_{k}, x\right\rangle-2^{-L+1}-\cdots-2^{-L+k-1} \geq 3 / 4-2^{-L+k} \geq 1 / 2 .
\end{aligned}
$$

Hence the inequality;

$$
\left|\left\langle u_{k}, x\right\rangle^{2}-\left\langle u_{k}, y\right\rangle^{2}\right|=\left|\sum_{1}^{k-1}\left(\left\langle u_{i}, x\right\rangle^{2}-\left\langle u_{i}, y\right\rangle^{2}\right)\right| \leq 2 \sum_{1}^{k-1}\left\langle u_{i}, x-y\right\rangle \mid
$$

implies

$$
\left|\left\langle u_{k}, x-y\right\rangle\right| \leq 2 \sum_{1}^{k-1}\left|\left\langle u_{i}, x-y\right\rangle\right|,
$$

and this yields that

$$
\begin{aligned}
\|x-y\| & \leq \sum_{1}^{k}\left|\left\langle u_{i}, x-y\right\rangle\right| \leq 3 \sum_{1}^{k-1}\left(1+2^{L+i+1}\right)^{2} \alpha \\
& \leq 3\left((k-1)+2^{-L+k+3}\right) \alpha .
\end{aligned}
$$

Q.E.D.

From now we return to the situation in Lemma 2.1'. Let $\left\{x_{i}\right\}$ be a $2^{-(n+7)} r$-dense and $2^{-(n+10)} r$-discrete subset of $B(0, r)$ and let $\left\{y_{i}\right\}$ be a system of points in $B(0, r)$ with $y_{1}=0$ such that

$$
(1+\varepsilon)^{-1} \leq\left\|y_{i}-y_{j}\right\| /\left\|x_{i}-x_{j}\right\| \leq 1+\varepsilon \text { for every } i \neq j .
$$

LEMMA 2.6. $\left|\Varangle\left(x_{i}, x_{j}\right)-\Varangle\left(y_{i}, y_{j}\right)\right| \leq 2^{(n / 2)+8} \varepsilon^{1 / 2}$ for every $i \neq j$.

Proof. Set $\alpha_{i, j}:=\Varangle\left(x_{i}, x_{j}\right)$ and $\beta_{i, j}:=\Varangle\left(y_{i}, y_{j}\right)$. First we show that $\left|\cos \alpha_{i, j}-\cos \beta_{i, j}\right| \leq 2^{(n+13)} \varepsilon$. Set $\kappa=1+\varepsilon$, then we get

$$
\begin{aligned}
\cos \alpha_{i, j} & =\left(\left\|x_{i}\right\|^{2}+\left\|x_{j}\right\|^{2}-\left\|x_{i}-x_{j}\right\|^{2}\right) / 2\left\|x_{i}\right\|\left\|x_{j}\right\| \\
& \leq\left(\kappa^{2}\left(\left\|y_{i}\right\|^{2}+\left\|y_{j}\right\|^{2}\right)-\kappa^{-2}\left\|y_{i}-y_{j}\right\|^{2}\right) / 2\left\|x_{i}\right\|\left\|x_{j}\right\| \\
& =\left(\kappa^{2}\left(2\left\|y_{i}\right\|\left\|y_{j}\right\| \cos \beta_{i, j}+\left\|y_{i}-y_{j}\right\|^{2}\right)-\kappa^{-2}\left\|y_{i}-y_{j}\right\|^{2}\right) / 2\left\|x_{i}\right\|\left\|x_{j}\right\| \\
& =\kappa^{2} \cos \beta_{i, j} \cdot\left\|y_{i}\right\|\left\|y_{j}\right\| /\left\|x_{i}\right\|\left\|x_{j}\right\|+\left(\kappa^{2}-\kappa^{-2}\right)\left\|y_{i}-y_{j}\right\|^{2} / 2\left\|x_{i}\right\|\left\|x_{j}\right\| \\
& \leq \kappa^{4} \cos \beta_{i, j}+\left(\kappa^{2}-\kappa^{-2}\right)\left(2^{(n+10)} \kappa+\kappa^{2}\right),
\end{aligned}
$$




$$
\begin{aligned}
\cos \alpha_{i, j}-\cos \beta_{i, j} & \leq\left(\kappa^{4}-1\right) \cos \beta_{i, j}+\left(\kappa^{2}-\kappa^{-2}\right)\left(2^{(n+10)} \kappa+\kappa^{2}\right) \\
& \leq 2^{(n+13)} \varepsilon:
\end{aligned}
$$

Hence we can get that $\left|\cos \alpha_{i, j}-\cos \beta_{i, j}\right| \leq 2^{(n+13)} \varepsilon$, and this yields

$$
\begin{aligned}
& 2\left(\sin \left(\left|\alpha_{i, j}-\beta_{i, j}\right| / 2\right)\right)^{2} \leq 2^{(n+13)} \varepsilon \\
& \left|\alpha_{i, j}-\beta_{i, j}\right| \leq 2 \sin ^{-1}\left(\left(2^{n+12} \varepsilon\right)^{1 / 2}\right) \\
& \quad \leq 2^{(n / 2)+8} \varepsilon^{1 / 2} \quad\left(\varepsilon \leq 2^{-(n+14)}\right) .
\end{aligned}
$$

Q.E.D.

Lemma 2.7. There exist $\left\{x_{m_{j}}\right\}_{1 \leq j \leq n} \subset\left\{x_{i}\right\}$ and $\left\{y_{m_{j}}\right\}_{1 \leq j \leq n} \subset\left\{y_{i}\right\}$ such that they are $\left(r, 2^{-(n+4)}\right)$-normal systems for $\boldsymbol{R}^{n}$.

Proof. Take an orthogonal basis $\left\{w_{j}\right\}$ for $\boldsymbol{R}^{n}$ such that $\left\|w_{j}\right\|=$ $\left(1-2^{-(n+6)}\right) r$, and by denseness, take $\left\{x_{m_{j}}\right\}_{1 \leq j \leq n} \subset\left\{x_{i}\right\}$ such that $\left\|x_{m_{j}}-w_{j}\right\|$ $\leq 2^{-(n+7)} r$. An easy calculation shows that $\left\{X_{m_{j}}\right\}_{1 \leq j \leq n}$ and the corresponding $\left\{y_{m_{j}}\right\}_{1 \leq j \leq n}$ have the required properties.

Q.E.D.

Proof of Lemma 2.1'. Let $\left\{u_{i}\right\}$ and $\left\{v_{i}\right\}$ be the orthonormal bases for $\boldsymbol{R}^{n}$ obtained by applying the Gram-Schmidt process to $\left\{x_{m_{i}}\right\}$ and $\left\{y_{m_{i}}\right\}$ respectively. A required linear isometry $I$ of $\boldsymbol{R}^{n}$ is defined by $I\left(u_{i}\right):=v_{i}$. If we set $X_{k}=I\left(x_{m_{k}}\right) /\left\|I\left(x_{m_{k}}\right)\right\|$ and $Y_{k}=y_{m_{k}} /\left\|y_{m_{k}}\right\|$, then we have with Lemma $2.3(1)$

$$
\left\langle v_{k}, X_{k}\right\rangle,\left\langle v_{k}, Y_{k}\right\rangle \geq 1-2^{-(n+4-k)} .
$$

This yields

$$
\begin{aligned}
\left\langle X_{k}, Y_{k}\right\rangle & \geq \cos \left(\Varangle\left(X_{k}, v_{k}\right)+\Varangle\left(v_{k}, Y_{k}\right)\right) \\
& \geq 2 \cos ^{2} \theta-1 \quad\left(\cos \theta:=1-2^{-(n+4-k)}\right) \\
& \geq 1-2^{-(n+2-k)} \geq 3 / 4 .
\end{aligned}
$$

ASSERTION 1. $\Varangle\left(I\left(x_{m_{k}}\right), y_{m_{k}}\right) \leq(6 k-5) 6^{k-2}(k-1) ! \varepsilon^{\prime} . \quad \varepsilon^{\prime}:=2^{(n / 2)+8} \varepsilon^{1 / 2}$.

Proof. From the triangle inequality and Lemma 2.6, we have

$$
\begin{aligned}
\Varangle\left(y_{m_{i}}, I\left(x_{m_{k}}\right)\right) & \leq \Varangle\left(I\left(x_{m_{k}}\right), I\left(x_{m_{i}}\right)\right)+\Varangle\left(I\left(x_{m_{i}}\right), y_{m_{i}}\right) \\
& \leq \Varangle\left(y_{m_{k}}, y_{m_{i}}\right)+\Varangle\left(I\left(x_{m_{i}}\right), y_{m_{i}}\right)+\varepsilon^{\prime},
\end{aligned}
$$

and similarly,

$$
\Varangle\left(y_{m_{i}}, I\left(x_{m_{k}}\right)\right) \geq \Varangle\left(y_{m_{k}}, y_{m_{i}}\right)-\Varangle\left(I\left(x_{m_{i}}\right), y_{m_{i}}\right)-\varepsilon^{\prime},
$$

hence,

$$
\left|\Varangle\left(y_{m_{i}}, I\left(x_{m_{k}}\right)\right)-\Varangle\left(y_{m_{i}}, y_{m_{k}}\right)\right| \leq \Varangle\left(I\left(x_{m_{i}}\right), y_{m_{i}}\right)+\varepsilon^{\prime} .
$$


Clearly, $\Varangle\left(I\left(x_{m_{1}}\right), y_{m_{1}}\right)=0$. Assume the assertion for $i, 1 \leq i \leq k-1$, then we get for every $i(1 \leq i \leq k-1)$

$$
\begin{aligned}
\left|\Varangle\left(y_{m_{i}}, I\left(x_{m_{k}}\right)\right)-\Varangle\left(y_{m_{i}}, y_{m_{k}}\right)\right| & \leq(6 i-5) 6^{i-2}(i-1) ! \varepsilon^{\prime}+\varepsilon^{\prime} \\
& \leq\left((6 k-11) 6^{k-3}(k-2) !+1\right) \varepsilon^{\prime} .
\end{aligned}
$$

Notice that $\left\{y_{m_{i}} /\left\|y_{m_{i}}\right\|\right\}_{1 \leq i \leq k}$ is a $\left(1,2^{-(n+3)}\right)$-normal system for its spanning subspace. Hence applying Lemma 2.5 to $\left\{y_{m_{i}} /\left\|y_{m_{i}}\right\|\right\}_{1 \leq i \leq k}, X_{k}$ and $Y_{k}$ in place of $\left\{e_{i}\right\}_{1 \leq i \leq k}, x$ and $y$, we conclude

$$
\Varangle\left(I\left(x_{m_{k}}\right), y_{m_{k}}\right) \leq(6 k-5) 6^{k-2}(k-1) ! \varepsilon^{\prime} . \quad \text { Q.E.D. }
$$

Assertion 2. $\left|\left\|I\left(x_{i}\right)-y_{m_{k}}\right\|-\left\|y_{i}-y_{m_{k}}\right\|\right| \leq\left(2 k ! 6^{k} \varepsilon^{\prime}\right)^{1 / 2} r$

for every $i$ and every $k, 1 \leq k \leq n$.

This and Lemma 2.4 complete the proof of Lemma 2.1'.

Proof of Assertion 2. Assertion 1 and the triangle inequality imply that

$$
\begin{aligned}
\Varangle\left(I\left(x_{i}\right), y_{m_{k}}\right) & \leq \Varangle\left(I\left(x_{i}\right), I\left(x_{m_{k}}\right)+\Varangle\left(I\left(x_{m_{k}}\right), y_{m_{k}}\right)\right. \\
& \leq \Varangle\left(y_{i}, y_{m_{k}}\right)+\left((6 k-5) 6^{k-2}(k-1) !+1\right) \varepsilon^{\prime},
\end{aligned}
$$

and similarly,

$$
\Varangle\left(I\left(x_{i}\right), y_{m_{k}}\right) \geq \Varangle\left(y_{i}, y_{m_{k}}\right)-\left((6 k-5) 6^{k-2}(k-1) !+1\right) \varepsilon^{\prime},
$$

hence,

$$
\left|\Varangle\left(I\left(x_{i}\right), y_{m_{k}}\right)-\Varangle\left(y_{i}, y_{m_{k}}\right)\right| \leq\left((6 k-5) 6^{k-2}(k-1) !+1\right) \varepsilon^{\prime} .
$$

Therefore we have

$$
\begin{aligned}
& \left|\left\|I\left(x_{i}\right)-y_{m_{k}}\right\|^{2}-\left\|y_{i}-y_{m_{k}}\right\|^{2}\right| \\
& \quad \leq\left|\left\|I\left(x_{i}\right)\right\|^{2}-\left\|y_{i}\right\|^{2}\right|+2\left\|y_{m_{k}}\right\| \mid\left\|y_{i}\right\| \cos \Varangle\left(y_{i}, y_{m_{k}}\right) \\
& \quad-\left\|I\left(x_{i}\right)\right\| \cos \Varangle\left(I\left(x_{i}\right), y_{m_{k}}\right) \mid,
\end{aligned}
$$

where $\left|\left\|I\left(x_{i}\right)\right\|^{2}-\left\|y_{i}\right\|^{2}\right| \leq 2 \varepsilon r^{2}$ and

$$
\begin{aligned}
\mid\left\|y_{i}\right\| & \cos \Varangle\left(y_{i}, y_{m_{k}}\right)-\left\|I\left(x_{i}\right)\right\| \cos \Varangle\left(I\left(x_{i}\right), y_{m_{k}}\right) \mid \\
& \leq r\left(\mid \Varangle\left(y_{i}, y_{m_{k}}\right)-\Varangle\left(I\left(x_{i}\right), y_{m_{k}}\right)+\varepsilon\right) \\
& \leq\left((6 k-5) 6^{k-2}(k-1) !+2\right) \varepsilon^{\prime} r .
\end{aligned}
$$

Hence the inequality

$$
\left|\left\|I\left(x_{i}\right)-y_{m_{k}}\right\|-\left\|y_{i}-y_{m_{k}}\right\|\right| \leq\left|\left\|I\left(x_{i}\right)-y_{m_{k}}\right\|^{2}-\left\|y_{i}-y_{m_{k}}\right\|^{2}\right|^{1 / 2}
$$

implies the required estimate.

Q.E.D. 


\section{§3. Reduction and $C^{0}$-estimates}

In this section we average the local diffeomorphisms $F_{i}$, constructed in the previous section, with a center of mass technique to obtain a smooth map $F: M \rightarrow \bar{M}$ and control the $C^{0}$ error between $F$ and $F_{i}$. Let $\psi$ be a smooth function such that

$$
\psi|[0,4]=1, \quad \psi|[5, \infty)=0, \quad 0 \geq \psi^{\prime} \geq-2 .
$$

For every $x \in M$, define the weights $\phi_{i}(x)$ of $F_{i}(x)$ by

$$
\phi_{i}(x):=\psi\left(d\left(x, p_{i}\right) / r\right) / \sum_{j} \psi\left(d\left(x, p_{j}\right) / r\right) .
$$

Notice that all $p_{j}$ with $d\left(x, p_{j}\right) \leq 5 r$ are finite and the corresponding $F_{j}(x)$ are contained in some convex ball $B$. It is easy from convexity argument to see that for a fixed $x \in M$, the function $C_{x}: \bar{M} \rightarrow \boldsymbol{R}$ defined by $C_{x}(y)=\frac{1}{2} \sum_{i} \phi_{i}(x) d^{2}\left(y, F_{i}(x)\right)$ is $C^{\infty}$ strongly convex on $B$, and has a unique minimum point on $\bar{M}$. Setting

$$
F(x):=\text { the unique minimum point of } C_{x}
$$

we define a map $F: M \rightarrow \bar{M}$. We show that $F$ is smooth. Define a map $V$ from a sufficiently small neighborhood of the graph of $F$ in $M \times \bar{M}$ to the tangent bundle $T \bar{M}$ by

$$
V(x, y):=-\sum_{i} \phi_{i}(x) \exp _{y}^{-1}\left(F_{i}(x)\right) .
$$

Since $V(x, y)=\left(\operatorname{grad} C_{x}\right)(y)$, we have $V(x, F(x))=0$. Let $K: T T \bar{M} \rightarrow T \bar{M}$ be the connection map, and define a map $D_{2} V_{(x, y)}: \bar{M}_{y} \rightarrow \bar{M}_{y}$ by $D_{2} V_{(x, y)}(\dot{y}(0))$ $=\nabla_{\dot{y}(0)} V(x, y(t))$, where we consider $V(x, y(t))$ as a vector field along a smooth curve $y(t)$ with $\dot{y}(0)=y$. Notice that

$$
K\left(d /\left.d t V(x, y(t))\right|_{t=0}\right)=D_{2} V_{(x, y)}(\dot{y}(0)),
$$

and $D_{2} V_{(x, y)}$ is a linear map. From the standard Jacobi fields estimates (See (4.3) in the proof of Lemma 4.2),

$$
\left\|D_{2} V_{(x, y)}(\dot{y}(0))-\dot{y}(0)\right\| \leq(30 \Lambda r)^{2}\|\dot{y}(0)\|<\|\dot{y}(0)\| .
$$

This yields that $D_{2} V_{(x, y)}$ is a linear isomorphism, and hence for $y=F(x)$, the space spanned by $\left\{d /\left.d t V(x, y(t))\right|_{t=0}\right\}$ and the (horizontal) tangent space of the zero section of $T \bar{M}$ at $(F(x), 0)$ span $(T \bar{M})_{(F(x), 0)}$. Therefore the implicit function theorem implies the smoothness of $F$.

From now on we fix $x_{0} \in M$ and set $y_{0}:=F\left(x_{0}\right)$. 
LEMMA 3.1. $d F_{x_{0}}$ has maximal rank iff

(*)

$$
\begin{aligned}
\sum_{i} d /\left.d t \psi\left(d\left(x(t), p_{i}\right) / r\right)\right|_{t=0} \cdot \exp _{y_{0}}^{-1}\left(F_{i}\left(x_{0}\right)\right) \\
\quad+\sum_{i} \psi\left(d\left(x_{0}, p_{i}\right) / r\right) \cdot d\left(\exp _{y_{0}}^{-1}\right)\left(d F_{i}(\dot{x}(0))\right) \neq 0
\end{aligned}
$$

for every smooth curve $x(t)$ with $x(0)=x_{0}$ and $\dot{x}(0) \neq 0$.

Proof. Differentiating the curve $V(x(t), F(x(t)))$ in the zero section of $T \bar{M}$, we have

$$
d /\left.d t V\left(x(t), y_{0}\right)\right|_{t=0}+D_{2} V_{\left(x_{0}, y_{0}\right)}(d F(\dot{x}(0)))=0 .
$$

Hence $d F_{x_{0}}$ has maximal rank iff $d /\left.d t V\left(x(t), y_{0}\right)\right|_{t=0} \neq 0$. Since $V\left(x_{0}, y_{0}\right)=0$,

$$
\begin{array}{rl}
d / d t & \left.V\left(x(t), y_{0}\right)\right|_{t=0} \\
= & -\sum_{i} d /\left.d t \psi_{i}\left(d\left(x(t), p_{i}\right) / r\right)\right|_{t=0} \cdot \exp _{y_{0}}^{-1}\left(F_{i}\left(x_{0}\right)\right) / \sum_{j} \psi_{j}\left(d\left(x_{0}, p_{j}\right) / r\right) \\
& \quad-\sum_{i} \phi_{i}\left(x_{0}\right) \cdot d\left(\exp _{y_{0}}^{-1}\right)\left(d F_{i}(\dot{x}(0))\right)
\end{array}
$$

This completes the proof.

Q.E.D.

We will show in Section 4 that in the above $(*)$, the norm of the first term is smaller than that of the second if $r$ and $\varepsilon$ are taken sufficiently small. To do this we must first estimate the numbers of the sum in each term.

LEMMA 3.4. If $N_{1}:=\#\left\{i ; \psi\left(d\left(x_{0}, p_{i}\right) / r\right)=1\right\}$ and $N_{2}:=\#\left\{i: \psi\left(d\left(x_{0}, p_{i}\right) / r\right)\right.$ $\neq 0\}$, then $N_{2} / N_{1} \leq 6^{n}$.

Proof. Since $\left\{p_{i}\right\}$ is $2^{-(n+8)} r$-dense, the union of $B\left(p_{i}, 2^{-(n+8)} r\right)$ with $d\left(x_{0}, p_{i}\right) \leq 4 r$ covers the $3.9 r$-ball around $x_{0}$, and since $\left\{p_{i}\right\}$ is $2^{-(n+9)} r$ discrete, the family of $B\left(p_{i}, 2^{-(n+10)} r\right)$ with $d\left(x_{0}, p_{i}\right) \leq 5 r$ are disjoint and contained in the 5.1r-ball around $x_{0}$. It follows from the Rauch comparison theorem that

$$
N_{1} \geq \tilde{v}(3.9 r) / v\left(2^{-(n+8)} r\right), \quad N_{2} \leq v(5.1 r) / \tilde{v}\left(2^{-(n+10)} r\right) .
$$

Hence we can get an explicit bound for $N_{2} / N_{1}$.

Q.E.D.

Now we fix $i$ and $k$ such that $d\left(x_{0}, p_{i}\right), d\left(x_{0}, p_{k}\right) \leq 5 r$, and estimate $d\left(F_{i}\left(x_{0}\right), F_{k}\left(x_{0}\right)\right)$.

Lemma 3.5. $\left|d\left(q_{j}, F_{k}\left(x_{0}\right)\right)-d\left(q_{j}, F_{i}\left(x_{0}\right)\right)\right| \leq \delta_{2}$ for every $j$ with $d\left(p_{i}, p_{j}\right)$, $d\left(p_{k}, p_{j}\right) \leq 10 r$, where $\delta_{2}=2\left(\delta_{1}+600 \Lambda r\right)$. 
Proof. Notice that

$$
e^{-201 r} \leq d\left(F_{k}\left(x_{0}\right), F_{k}\left(p_{j}\right)\right) / d\left(x_{0}, p_{j}\right) \leq e^{201 r} .
$$

By Lemma 2.1,

$$
\left|d\left(q_{j}, F_{k}\left(x_{0}\right)\right)-d\left(F_{k}\left(p_{j}\right), F_{k}\left(x_{0}\right)\right)\right| \leq \delta_{1} r .
$$

Hence the triangle inequality implies

$$
\left|d\left(p_{j}, x_{0}\right)-d\left(q_{j}, F_{k}\left(x_{0}\right)\right)\right| \leq\left(\delta_{1} r+40 \Lambda r \cdot d\left(p_{j}, x_{0}\right)\right) \leq \delta_{2} r / 2 .
$$

From the same estimate for $i$, we have the required bound.

Q.E.D.

Here we assume the following bound on $\varepsilon$ and $r$ in order to bound $\delta_{2} \leq 1 / 2$

$$
\varepsilon, 20 \Lambda r \leq 2^{-18}(n+1)^{-4}\left(6^{n+2} n ! 2^{(n / 2)+7}\right)^{-2} .
$$

This bound assures that $d\left(F_{i}\left(x_{0}\right), F_{k}\left(x_{0}\right)\right) \leq 2 r / 3$.

LEMMA 3.7. $d\left(F_{k}\left(x_{0}\right), F_{i}\left(x_{0}\right)\right) \leq \delta_{3} r$, where $\delta_{3}=8(n+1) \delta_{2}$.

Proof. Take a $q_{m_{0}} \in\left\{q_{i}\right\}$ such that $d\left(q_{m_{0}}, F_{k}\left(x_{0}\right)\right) \leq 2^{-(n+8)} r$, and let $x_{k}$ and $x_{i}$ denote the images of $F_{k}\left(x_{0}\right)$ and $F_{i}\left(x_{0}\right)$ by $\exp _{q_{m_{0}}}^{-1}$ Then from the above bound (**) we have that $\left\|x_{k}\right\|,\left\|x_{i}\right\| \leq r$. By Lemma 2.7, we can choose $\left\{q_{m_{j}}\right\}_{1 \leq j \leq n}$ out of $\left\{q_{i}\right\}$ such that if $\tilde{q}_{m_{j}}$ denotes the image of $q_{m_{j}}$ by $\exp _{q_{m_{0}}}^{-1}$, then $\left\{\tilde{q}_{m_{j}}\right\}_{1 \leq j \leq n} 1$ is an $\left(r, 2^{-(n+4)}\right)$ normal system for $\bar{M}_{q_{m_{0}}}$. Notice that $\left\{p_{m_{j}}\right\}_{1 \leq j \leq n}$ corresponding to $\left\{q_{m_{j}}\right\}_{1 \leq j \leq n}$ are contained in $B\left(p_{k}, 10 r\right) \cap$ $B\left(p_{i}, 10 r\right)$. From Lemma 3.5 we have

$$
\left\|\tilde{q}_{m_{j}}-x_{k}\right\|-\left\|\tilde{q}_{m_{j}}-x_{i}\right\| \mid \leq 2 \delta_{2} r, \quad 0 \leq j \leq n,
$$

and together with Lemma 2.4 this yields

$$
d\left(F_{k}\left(x_{0}\right), F_{i}\left(x_{0}\right)\right) \leq 8(n+1) \delta_{2} r .
$$

From the definition of $F$ it is clear that $d\left(F\left(x_{0}\right), F_{i}\left(x_{0}\right)\right) \leq \delta_{3} r$ for every $i$ with $d\left(x_{0}, p_{i}\right) \leq 5 r$. Hence we have with Lemma 3.4

$$
\begin{array}{r}
\left\|\sum_{i} d /\left.d t \psi\left(d\left(x(t), p_{i}\right) / r\right)\right|_{t=0} \cdot \exp _{y_{0}}^{-1}\left(F_{i}\left(x_{0}\right)\right)\right\| \\
\leq N_{2}(2 / r) \delta_{3} r\|\dot{x}(0)\| \leq 2 \cdot 6^{n} \delta_{3} N_{1}\|\dot{x}(0)\| .
\end{array}
$$

\section{§4. $C^{1}$-estimates}

To estimate the second term in Lemma $3.1(*)$ from below, we must control the error between $d\left(\exp _{y_{0}}^{-1}\right)\left(d F_{i}(\dot{x}(0))\right)$ and $d\left(\exp _{y_{0}}^{-1}\right)\left(d F_{k}(\dot{x}(0))\right)$. To 
do this it is essential to estimate $\left\|d F_{k}(\dot{x}(0))-P d F_{i}(\dot{x}(0))\right\|$ from above, where $P$ denotes the parallel translation along the minimizing geodesic from $F_{i}\left(x_{0}\right)$ to $F_{k}\left(x_{0}\right)$. This is done in Lemma 4.5.

Lemma 4.1. For each $x \in \bar{M}$, let $\left\{q_{\alpha_{j}}\right\}:=\left\{q_{i}\right\} \cap B(x, r)$ and $N^{\prime}:=\#\left\{q_{\alpha_{i}}\right\}$. The map $\Phi: B(x, r / 2) \rightarrow \boldsymbol{R}^{N^{\prime}}$ defined by $\Phi^{j}(y)=d^{2}\left(q_{\alpha_{j}}, y\right)$ satisfies the following;

(1) $\Phi$ is an embedding, and $\|d \Phi(v)\| \geq r\|v\|$ for every tangent vector $v$ on $B(x, r / 2)$,

(2) $\quad N^{\prime} \leq 2^{n(n+11)}$.

Proof. The convexity of each component $\Phi^{i}$ of $\Phi$ implies the injectivity of $\Phi$. For a given tangent vector $v$ on $B(x, r / 2)$, let $r$ be a geodesic with $\dot{\gamma}(0)=v /\|v\|$. Take a $q_{\alpha j}$ such that $d\left(q_{\alpha_{j}}, \gamma(r / 2)\right) \leq 2^{-(n+8)} r$. Comparing the triangle with vertices $\left(\gamma(0), \gamma(r / 2), q_{\alpha_{j}}\right)$ to a triangle with the same edge length in the sphere with constant curvature $\Lambda^{2}$, we have that cos $\Varangle(\dot{\gamma}(0), \dot{\sigma}(0)) \geq 1 / 2$, where $\sigma$ denote a unique minimizing geodesic from $r(0)$ to $q_{\alpha_{j}}$. This yields that

$$
\|d \Phi(v)\| \geq\left|d \Phi^{j}(v)\right| \geq r\|v\| .
$$

The same proof as in Lemma 3.4 implies (2).

Q.E.D.

We fix $i$ and $k$ with $d\left(p_{i}, x_{0}\right), d\left(p_{k}, x_{0}\right) \leq 5 r$ and take an embedding $\Phi: B\left(F_{k}\left(x_{0}\right), r / 2\right) \rightarrow \boldsymbol{R}^{N^{\prime}}$ defined in the previous lemma for $F_{k}\left(x_{0}\right)$, where we set $\left\{q_{\alpha_{j}}\right\}:=\left\{q_{i}\right\} \cap B\left(F_{k}\left(x_{0}\right), r\right)$. For a unit tangent vector $v$ at $x_{0}$, let $\gamma, \sigma_{k}$ and $\sigma_{i}$ be geodesics such that $\dot{\gamma}(0)=v, \dot{\sigma}_{k}(0)=d F_{k}(v)$ and $\dot{\sigma}_{i}(0)=d F_{i}(v)$. For every $q_{\alpha j}$, we set

$$
\begin{array}{ll}
f_{j}(t)=d^{2}\left(p_{\alpha_{j}}, \gamma(t)\right), & g_{m, j}(t)=\Phi^{j}\left(F_{m} \cdot \gamma(t)\right), \\
h_{m, j}(t)=\Phi^{j}\left(\sigma_{m}(t)\right), & m=k, i .
\end{array}
$$

LEMMA 4.2. On $[0, r / 2]$,

(1) $2\left(1-\Lambda^{2} f_{j}\right) \leq f_{j}^{\prime \prime} \leq 2\left(1+\Lambda^{2} f_{j}\right)$,

$$
2\left(1-\Lambda^{2} h_{m, j}\right) e^{-20 \Lambda r} \leq h_{m, j}^{\prime \prime} \leq 2\left(1+\Lambda^{2} h_{m, j}\right) e^{20 \Lambda r}
$$

(2) $\left|g_{m, j}^{\prime \prime}-h_{m, j}^{\prime \prime}\right| \leq \Omega_{1} r$

where $\Omega_{1}=82+10 n^{3} \Omega r$,

$$
\Omega=60 n(n-1)\left(10 \Lambda_{1} r^{2}+4 \Lambda^{2} r+400 n^{3 / 2} \Lambda(\Lambda r)^{3}\right) e^{10\left(2 n^{2} \Lambda^{2}+1\right) r} .
$$

(2) is the only place where we need the assumption for $\|\nabla R\|$.

Proof. We consider geodesic veriations 


$$
\begin{aligned}
& \alpha(t, s)=\exp _{q_{\alpha_{j}}} s\left(\exp _{q_{\alpha_{j}}}^{-1} F_{m}(\gamma(t))\right), \\
& \beta(t, s)=\exp _{q_{\alpha_{j}}} s\left(\exp _{q_{\alpha_{j}}}^{-1} \sigma_{m}(t)\right) .
\end{aligned}
$$

Then for a fixed $t$, we have Jacobi fields

$$
J_{0}(s)=\frac{\partial \alpha}{\partial t}(t, s) \text { and } \quad J(s)=\frac{\partial \beta}{\partial t}(t, s),
$$

and the second variation formula yields

$$
g_{m, j}^{\prime \prime}(t)=2\left(\left\langle\nabla_{j_{0}} J_{0}, T_{0}\right\rangle+\left\langle J_{0}, \nabla_{T_{0}} J_{0}\right\rangle\right)(1), \quad h_{m, j}^{\prime \prime}(t)=2\left\langle J, \nabla_{T} J\right\rangle(1),
$$

where $T_{0}$ and $T$ denote the vector fields $\partial \alpha / \partial s$ and $\partial \beta / \partial s$. We assert that

$$
\left(1-\Lambda^{2}\|T\|^{2}\right)\|J(1)\|^{2} \leq\left\langle J, \nabla_{T} J\right\rangle(1) \leq\left(1+\Lambda^{2}\|T\|^{2}\right)\|J(1)\|^{2},
$$

which implies (1). Let $\tau$ be a geodesic with $\|\dot{\tau}\|=\|T\|$ in the $n$-sphere $S$ with constant curvature $\Lambda^{2}$ and $I$ a linear isometry from $\bar{M}_{q_{\alpha_{j}}}$ to $S_{\tau(0)}$, and $W$ the vector field along $\tau$ defined by using the parallel translations along $\beta(t, \quad)$ and $\tau$ and $I$. Then a standard comparison argument implies

$$
\left\langle J, J^{\prime}\right\rangle(1)=I_{0}\left(J, J^{\prime}\right) \geq I_{0}(W, W) \geq I_{0}(V, V)=\left\langle V, V^{\prime}\right\rangle(1),
$$

where $I_{0}$ denote the index form and $V$ the Jacobi field along $\tau$ with $V(0)=0$ and $V(1)=W(1)$. It is easy to check that

$$
\begin{aligned}
& \|V(s)\|^{2}=s^{2}\left\|J^{T}(1)\right\|^{2}+\frac{\sin ^{2} \Lambda\|T\| s}{\sin ^{2} \Lambda\|T\|}\left(\|J(1)\|^{2}-\left\|J^{T}(1)\right\|^{2}\right), \\
& \left\langle V, V^{\prime}\right\rangle(1)=\left\|J^{T}(1)\right\|^{2}+\Lambda\|T\| \cot \Lambda\|T\| \cdot\left(\|J(1)\|^{2}-\left\|J^{T}(1)\right\|^{2}\right),
\end{aligned}
$$

where $J^{T}$ denote the tangential component of $J$. Hence we have that $\left\langle J, J^{\prime}\right\rangle(1) \geq\left(1-\Lambda^{2}\|T\|^{2}\right)\|J(1)\|^{2}$. Let $P$ be a parallel vector field along $\beta(t$,$) , then we get$

$$
\left|\left\langle J(s)-s J^{\prime}(s), P\right\rangle^{\prime}\right|=|s\langle R(T, J) T, P\rangle| \leq 2 \Lambda^{2}\|T\|^{2}\|J\| s .
$$

The integration implies

$$
\left\|J(1)-J^{\prime}(1)\right\| \leq \Lambda^{2}\|T\|^{2}\|J(1)\| .
$$

It follows

$$
\left|\left\langle J, J^{\prime}\right\rangle(1)\right| \leq\|J(1)\|\left\|J^{\prime}(1)\right\| \leq\left(1+\Lambda^{2}\|T\|^{2}\right)\|J(1)\|^{2} .
$$

For (2), we get with (*) 


$$
\begin{aligned}
\mathrm{g}_{m, j}^{\prime \prime}(t)-h_{m, j}^{\prime \prime}(t) \mid & \leq 2\left|\left\langle J_{0}, J_{0}^{\prime}\right\rangle(1)-\left\langle J, J^{\prime}\right\rangle(1)\right|+2\left|\left\langle\nabla_{J_{0}} J_{0}, T_{0}\right\rangle(1)\right| \\
& \leq e^{20 \Lambda r}\left(2+8 \Lambda^{2} r^{2}\right)-e^{-20 \Lambda r}\left(2-8 \Lambda^{2} r^{2}\right)+2\left\|\nabla_{J_{0}(1)} J_{0}\right\| \cdot 2.3 r \\
& \leq 82 \Lambda r+4.6 r\left\|\nabla_{J_{0}(1)} J_{0}\right\| .
\end{aligned}
$$

Let $\left\{e_{i}\right\}$ be an orthonormal basis for $M_{p_{m}}$ and $\left\{x_{i}\right\},\left\{y_{i}\right\}$ the normal coordinate systems on $B\left(p_{m}, 10 r\right), B\left(q_{m}, 10 r\right)$ based on $\left\{e_{i}\right\},\left\{I_{m}\left(e_{i}\right)\right\}$ respectively. Let $\Gamma_{i, j}^{k}$ and $\bar{\Gamma}_{i, j}^{k}$ be the Cristoffel symbols with respect to $\left\{x_{i}\right\}$ and $\left\{y_{i}\right\}$ and let $c:=F_{m} \circ \gamma$. Note that

$$
\begin{aligned}
\dot{c}: & =\sum_{i} \dot{c}_{i} \frac{\partial}{\partial y_{i}}, \quad \ddot{c}_{k}+\sum_{i, j} \Gamma_{i, j}^{k}(\gamma(t)) \dot{c}_{i} \dot{c}_{j}=0, \\
\nabla_{\dot{c}} \dot{c} & =\sum_{k}\left(\ddot{c}_{k}+\sum_{i j} \bar{\Gamma}_{i, j}^{k}(c(t)) \dot{c}_{i} \dot{c}_{j}\right) \frac{\partial}{\partial y_{k}} \\
& =\sum_{k, i, j}\left(-\Gamma_{i, j}^{k}(\gamma(t))+\bar{\Gamma}_{i, j}^{k}(c(t))\right) \dot{c}_{i} \dot{c}_{j} \frac{\partial}{\partial y_{k}} .
\end{aligned}
$$

By the Rauch comparison theorem, we get

$$
\begin{aligned}
& \left|\dot{c}_{i}\right| \leq e^{10 \Lambda r}\|\dot{c}\| \leq e^{30 \Lambda r}, \quad\left\|\frac{\partial}{\partial y_{k}}\right\| \leq e^{10 \Lambda r}, \\
& \left|\Gamma_{i, j}^{k}\right| \leq e^{10 \Lambda r}\left\|\nabla_{\partial / \partial x_{i}} \frac{\partial}{\partial x_{j}}\right\|, \quad\left|\bar{\Gamma}_{i, j}^{k}\right| \leq e^{10 \Lambda r}\left\|\nabla_{\partial / \partial y_{i}} \frac{\partial}{\partial y_{j}}\right\|,
\end{aligned}
$$

and from a Cheeger's result (See [4], Lemma 4.3), we can estimate with $(* *)$ in Section 3

$$
\left\|\nabla_{\partial / \partial x_{i}} \frac{\partial}{\partial x_{j}}\right\|, \quad\left\|\nabla_{\partial / \partial y_{i}} \frac{\partial}{\partial y_{j}}\right\| \leq \Omega .
$$

Therefore we conclude that $\left\|\nabla_{\dot{c}} \dot{c}\right\| \leq 2 n^{3} e^{80 \Lambda r} \Omega$, and this yields (2). Q.E.D.

The following lemma is used in the proof of Lemma 4.5.

LEMMA 4.4. Let $\varphi ;[0, t] \rightarrow \boldsymbol{R}$ be a $C^{2}$-function such that $\varphi(0)=0$ and $|\varphi(s)| \leq \alpha,\left|\varphi^{\prime \prime}(s)\right| \leq \kappa$ on $[0, t]$. Then $\left|\varphi^{\prime}(0)\right| \leq \alpha / t+\kappa t / 2$.

LEMMA 4.5. $\left\|P d F_{i}(v)-d F_{k}(v)\right\| \leq 2^{n(n+11) / 2}\left(11 \delta_{3}+\Omega_{1} r / 2\right)$, where $P d e-$ notes the parallel translation along the minimizing geodesic from $F_{i}\left(x_{0}\right)$ to $F_{k}\left(x_{0}\right)$.

Proof. Let $\tau$ be a geodesic with $\dot{\tau}(0)=P d F_{i}(v)$ and let $u_{j}(t):=\Phi^{j}(\tau(t))$. We apply the previous lemma to $h_{k, j}-u_{j}$. On [0,r/2] we have with (3.6) and Lemma 4.2 (2) 


$$
\begin{aligned}
\left|h_{k, j}-h_{i, j}\right| & \leq\left|h_{k, j}-g_{k, j}\right|+\left|g_{k, j}-f_{j}\right|+\left|f_{j}-g_{i, j}\right|+\left|g_{i, j}-h_{i, j}\right| \\
& \leq 4 \delta_{2} r^{2}+\Omega_{1} r^{3} 4 .
\end{aligned}
$$

and the Rauch comparison theorem implies

$$
\left|h_{i, j}-u_{j}\right| \leq d\left(\sigma_{i}(0), \tau(0)\right) \cosh 4 r \cdot 4 r \leq 5 \delta_{3} r^{2},
$$

hence

$$
\left|h_{k, j}-u_{j}\right| \leq\left(4 \delta_{2}+5 \delta_{3}+\Omega_{1} r / 4\right) r^{2} .
$$

Together with Lemma 4.2 (1), Lemma 4.4 applied to $\varphi=h_{k, j}-u_{j}$ yields

$$
\begin{aligned}
\left|d \Phi^{j}\left(\dot{\sigma}_{k}(0)-\dot{\tau}(0)\right)\right| & \leq 2\left(4 \delta_{2}+5 \delta_{3}+\Omega_{1} r / 4\right) r+824 r^{2} / 4 \\
& \leq\left(11 \delta_{3}+\Omega_{1} r / 2\right) r .
\end{aligned}
$$

By Lemma 4.1, we conclude

$$
\left\|P d F_{i}(v)-d F_{k}(v)\right\| \leq 2^{n(n+11) / 2}\left(11 \delta_{3}+\Omega_{1} r / 2\right) .
$$

Let $P_{k}, P_{i}$ denote the parallel translation along the minimizing geodesics from $y_{0}$ to $F_{k}\left(x_{0}\right), F_{i}\left(x_{0}\right)$, and for simplicity, set

$$
v_{m}:=d F_{m}(v), \quad \tilde{v}_{m}:=d\left(\exp _{y_{0}}^{-1}\right)\left(d F_{m}(v)\right), \quad m=i, k .
$$

LEMMA 4.6. $\left\|\tilde{v}_{k}-\tilde{v}_{i}\right\| \leq \delta_{4}$, where $\delta_{4}=2^{n(n+11) / 2}\left(12 \delta_{3}+\Omega_{1} r / 2\right)$.

Proof. From standard estimate of the Jacobi equation and an easy comparison argument, we get

$$
\left\|P_{k} \tilde{v}_{k}-v_{k}\right\|, \quad\left\|P_{i}^{-1} v_{i}-\tilde{v}_{i}\right\|, \quad\left\|P v_{i}-P_{k} P_{i}^{-1} v_{i}\right\| \leq \Lambda^{2} r^{2} .
$$

Together with Lemma 4.5 , this yields

$$
\begin{aligned}
\left\|\tilde{v}_{k}-\tilde{v}_{i}\right\|= & \left\|P_{k} \tilde{v}_{k}-P_{k} \tilde{v}_{i}\right\| \\
\leq & \left\|P_{k} \tilde{v}_{k}-v_{k}\right\|+\left\|v_{k}-P v_{i}\right\|+\left\|P v_{i}-P_{k} P_{i}^{-1} v_{i}\right\| \\
& \quad+\left\|P_{k} P_{i}^{-1} v_{i}-P_{k} \tilde{v}_{i}\right\| \\
\leq & 2^{n(n+11) / 2}\left(12 \delta_{3}+\Omega_{1} r / 2\right) .
\end{aligned}
$$

Proof of Theorem 1. By Lemma 4.6, we have

$$
\left\|\sum_{i} \psi\left(d\left(x_{0}, p_{i}\right) / r\right) \tilde{v}_{i}-\sum_{i} \psi\left(d\left(x_{0}, p_{i}\right) / r\right) \tilde{v}_{k}\right\| \leq \delta_{4} N_{2},
$$

hence with Lemma 3.4

$$
\left\|\sum_{i} \psi\left(d\left(x_{0}, p_{i}\right) / r\right) \tilde{v}_{i}\right\| \geq\left(0.9-6^{n} \delta_{4}\right) N_{1} .
$$

If we set $\varepsilon \leq \varepsilon_{1}, r \leq r_{1}$, then we get with (3.8) 


$$
\left\|\sum_{i} \psi\left(d\left(x_{0}, p_{i}\right) / r\right) \tilde{v}_{i}\right\|>\left\|\sum_{i} d /\left.d t \psi\left(d\left(\gamma(t), p_{i}\right) / r\right)\right|_{t=0} \cdot \exp _{y_{0}}^{-1}\left(F_{i}\left(x_{0}\right)\right)\right\|+0.1 N_{1} .
$$

By Lemma 3.1, $F$ is an immersion. Furthermore the above inequality and (3.3) imply

$$
\left\|d /\left.d t V\left(\gamma(t), y_{0}\right)\right|_{t=0}\right\|>0.1 N_{1} / N_{2} \text {. }
$$

On the other hand, a standard Jacobi fields estimate (4.3) yields

$$
\left\|\nabla_{d F(v)} V\left(x_{0}, F(\gamma(t))\right)\right\| \leq 4 N_{2}\|d F(v)\| .
$$

Hence we have with (3.2) and Lemma 3.4

$$
\|d F(v)\| \geq N_{1} / 40 N_{2}^{2} \geq \tilde{v}\left(2^{-(n+10)} r\right) / 40 \cdot 6^{n} v(5.1 r)>0 .
$$

This conclude that $F$ must be surjective, and hence injective since it is a homotopy equivalence by its construction.

Q.E.D.

Added in proof. Recently we have received a preprint, S. Peters "Cheeger's finiteness theorem for diffeomorphism classes of Riemannian manifolds", where the finiteness of diffeomorphism classes of Cheeger type is proved for all dimensions without the assumption for $\|\nabla R\|$ by using a similar method to our Theorem 1.

\section{REFERENCES}

[1] R. Bishop and R. Crittenden, Geometry of manifolds, Academic Press, New-York, 1964.

[ 2 ] P. Buser and H. Karcher, Gromov's almost flat manifolds, Astérisque, 1981.

[ 3 ] J. Cheeger, Comparison and finiteness theorems for Riemannian manifolds, Ph. D. Thesis, Princeton Univ., 1967.

[ 4 ] _ Pinching theorems for a certain class of Riemannian manifolds, Amer. J. Math., 91 (1969), 807-834.

[ 5 ] — Finiteness theorems for Riemannian manifolds, Amer. J. Math., 92 (1970), 61-74.

[6] J. Cheeger and D. Ebin, Comparison theorems in Riemannian geometry, NorthHolland, 1975.

[ 7 ] R. Greene, Complete metrics of bounded curvature on noncompact manifolds, Arch. Math., 31 (1978), 89-95.

[ 8 ] M. Gromov, Almost flat manifolds, J. Differential Geom., 13 (1978), 231-241.

[ 9 ] — Structures métriques pour les variétés riemanniennes, rédigé par J. Lafontaine et P. Pansu, Cedic-Fernand Nathan, Paris, 1981.

[10] E. Heintz and H. Karcher, A general comparison theorem with applications to volume estimates for submanifolds, Ann. Sci. Ecole Norm. Sup., 11 (1978) , 451-470.

[11] M. Maeda, Volume estimate of submanifolds in compact Riemannian manifolds, J. Math. Soc. Japan, 30 (1978), 533-551.

[12] Y. Shikata, On a distance function on the set of differentiable structures, Osaka J. Math., 3 (1966), 65-79. 
[13] A. Weinstein, On the homotopy type of positively-pinched manifolds, Arch. Math., 18 (1967), 523-524.

Saga University

Faculty of Science and Engineering

Saga 840, Japan 